\title{
Iterative Model Reconstruction in Lumbar Spine Image Retrieval from Computed Tomography of the Abdomen and Pelvis
}

\author{
SY Tan ${ }^{1}$, A Kuganesan'1, K Buchan ${ }^{1}$, KK Lau ${ }^{1,2}$ \\ ${ }^{I}$ Diagnostic Imaging Department, Monash Health, Victoria, Australia \\ ${ }^{2}$ Faculty of Medicine, Nursing and Health Sciences, Monash University, Victoria, Australia
}

\begin{abstract}
Objective: We sought to compare the image quality of iterative model reconstruction (IMR) with statistical iterative reconstruction (SIR) in lumbar spine ( $L$-spine) images reconstructed from computed tomography $(C T)$ image data from examinations of the abdomen and pelvis compared with routine L-spine CT images reconstructed with SIR. Methods: We conducted a retrospective study to compare image noise in L-spine reconstructions using SIR and IMR techniques in consecutive CT abdomen and pelvis (CTAP) examinations, with L-spine CT images reconstructed using SIR. Hounsfield units and their standard deviations in areas of image noise were measured in bone, cerebrospinal fluid (CSF), and lumbar discs. The results of SIR and IMR were compared using paired $\mathrm{t}$ tests. The results of CTAP and CT L-spine were compared using the Mann-Whitney $\mathrm{U}$ test. A p value $<0.05$ was considered significant. Qualitative assessment of the bone/CSF, disc/CSF, and disc/bone interface was performed by two readers.

Results: IMR generated less image noise than SIR with CTAP and L-spine CT with SIR, particularly on bone windows. There was also significant improvement in the clarity of interfaces between vertebral bodies, discs, epidural fat, and CSF with IMR. The mean radiation dose was much higher for L-spine CT compared with CTAP.

Conclusion: IMR is superior to SIR for the image quality of reconstructed lumbar spine images from CTAP. The IMR lumbar spine image from CTAP image data acquired with a lower radiation dose was also shown to be clearer when compared with routine SIR L-spine CT images.
\end{abstract}

Key Words: Artifacts; Image processing, computer-assisted; Radiometry; Spine/diagnostic imaging; Tomography, X-ray computed

\footnotetext{
Correspondence: Dr SY Tan, Diagnostic Imaging Department, Monash Health, Victoria, Australia

Email: tanshuyi@gmail.com
}

Submitted: 1 Sep 2019; Accepted: 18 Nov 2019

Contributors: SYT and KKL designed the study. SYT acquired the data. SYT and KKL analysed the data. SYT drafted the manuscript. All authors critically revised the manuscript for important intellectual content. All authors had full access to the data, contributed to the study, approved the final version for publication, and take responsibility for its accuracy and integrity.

Conflicts of Interest: All authors have disclosed no conflicts of interest.

Funding/Support: This research received no specific grant from any funding agency in the public, commercial, or not-for-profit sectors.

Ethics Approval: This study was approved by the Monash Health Human Research Ethics Committee (Ref 16-0000-478Q), and the requirement for patient consent was waived. 


\title{
中文摘要
}

\section{迭代模型重建腹部和盆腔CT的腰椎圖像}

\author{
SY Tan 、A Kuganesan 、K Buchan 、 KK Lau \\ 目的 : 比較迭代模型重建 (IMR) 和統計迭代重建 (SIR) 腹部和盆腔CT的腰椎圖像質量, 並與常 \\ 規SIR 腰椎CT圖像質量作比較。 \\ 方法: 這項回顧性研究比較以SIR和IMR方法從連續腹部和盆腔CT重建出腰椎圖像的噪聲, 以及比 \\ 較SIR重建直接腰椎掃描圖像的噪聲, 測量骨、腦脊液和腰椎間盤的圖像噪聲（Hounsfield單位及其 \\ 標準差 $)$ 。使用配對 $t$ 檢驗比較SIR和IMR重建腹部和盆腔CT的結果, 以及使用Mann-Whitney $U$ 檢驗 \\ 比較腹部 / 盆腔CT與腰椎直接CT掃描的結果, $\mathrm{p}<0.05$ 為差異有統計學意義。由兩名閲片者對骨與 \\ 腦脊液、椎間盤與腦春液, 以及椎間盤與骨界面清晰度進行定性評估。
}

結果 : 相比SIR重建腹部和盆腔CT圖像和SIR重建腰椎CT圖像, IMR重建腹部和盆腔CT圖像的噪聲 較少, 尤以骨視窗測量為甚。透過IMR重建, 椎體、椎間盤、硬膜外脂肪和腦脊液間的界面清晰度 也得到顯著改善。與腹部和盆腔CT相比, 腰椎CT的平均放射劑量明顯較多。

結論 : 以IMR重建腹部和盆腔CT的腰椎圖像質量較SIR為佳。與常規SIR腰椎CT圖像相比, 以較低輻 射劑量採集腹部和盆腔CT圖像數據的IMR腰椎圖像更加清晰。

\section{INTRODUCTION}

Part of the process of optimising a radiographic examination involves reducing the radiation dose to as low as reasonably achievable whilst maintaining adequate image quality for diagnostic purposes. ${ }^{1}$ It is well known that reducing radiation dose increases image noise, and reduces image quality. ${ }^{2}$ Current radiology practice concentrates on techniques of dose reduction whilst optimising image quality. ${ }^{2}$

Computed tomography of the abdomen and pelvis (CTAP) has become the mainstay of a diagnostic workup in trauma settings, ${ }^{3}$ particularly if there is a high probability of an abdominal or pelvic injury. Lumbar spine reconstructions from CTAP datasets are frequently requested by emergency physicians for assessment of bony injury or pathology. 4,5 The image quality of the reconstructions is generally inferior to that of dedicated $\mathrm{CT}$ of the lumbar spine (L-spine CT). However, the better image quality of L-spine CT is at the expense of higher radiation dose and potentially missing other intraabdominal or pelvic pathology outside the field of view. ${ }^{6}$ Data from the Australian Radiation Protection and Nuclear Safety Agency have documented higher dose levels for CT lumbar spine (26 mGy) compared with CTAP (13 mGy). ${ }^{7}$
The rise of iterative reconstruction (IR) algorithms for $\mathrm{CT}$ in recent years has decreased the need for radiation dose increases in order to improve image quality. ${ }^{8,9}$

Filtered back projection was previously the most commonly used reconstruction algorithm for conventional CT imaging. It was associated with increased image noise in larger patients, but its advantage was a faster image reconstruction rate. ${ }^{10}$ In comparison, IR techniques generated better image quality with reduced image noise at lower radiation doses (though longer reconstruction times) compared with the filtered back projection technique. ${ }^{9,11}$ Dose reductions from IR techniques range widely in the available literature, ${ }^{11-14}$ some quoting as high as a $76 \%$ reduction in dose. ${ }^{15}$ This wide range of dose reduction is partially due to patient body habitus. Different models of IR techniques have been developed over the years, including statistical IR (SIR) and iterative model reconstruction (IMR). SIR utilises a set of algorithms to reduce the dose whereas IMR requires a more complex set of algorithms, taking into account the data statistics, image statistics, and system models. ${ }^{16}$ IMR generally takes a longer time to compute, ${ }^{17}$ which is usually offset by the faster processing capacities of current computers. 
IMR has been shown to reduce objective noise and improve subjective image quality. ${ }^{8}$ It also improves detection of small nodules on CT scans of the chest. ${ }^{18}$

Although there is a vast amount of literature available documenting the benefits of IR techniques, assessment of image quality improvement of the lumbar spine reconstruction from routine CTAP using IMR compared with SIR and its comparison with routine L-spine CT has not been documented in the current literature. This application of spinal reconstruction is particularly important in trauma and oncology settings.

The aim of this study was to determine the efficacy of IMR compared with SIR in image noise reduction on spinal reconstructions of CTAP and dedicated L-spine CT.

\section{METHODS}

\section{Study Design and Setting}

A retrospective study was performed on consecutive adult cases from the accident and emergency department who underwent CTAP, and another group of consecutive adult cases that underwent dedicated L-spine $\mathrm{CT}$ at the same tertiary hospital from July to September 2016.

\section{Scan Properties}

All patients had been scanned using a 256 multi-slice CT scanner (ipatient CT [iCT]; Philips Healthcare, Cleveland $[\mathrm{OH}]$, US) using the standard CTAP or L-spine CT protocol in our institution. All CTAPs were performed with intravenous contrast as per our institution's standard protocol, whereas L-spine CTs were performed without contrast. Lumbar spine reconstructions from CTAP image data were reconstructed in the axial, sagittal, and coronal planes with $3 \mathrm{~mm}$ section thickness using SIR (iDose4, Philips Healthcare, Cleveland [OH], US) and level 1 (lowest level) IMR (Philips Healthcare, Cleveland $[\mathrm{OH}], \mathrm{US})$ techniques. An average pitch of 1 was used at our centre.

Dedicated L-spine CT images were reconstructed with SIR in the axial, sagittal, and coronal planes with 3-mm section thickness.

Scanning parameters such as $\mathrm{kVp}$, mAs, volume CT dose index (CTDIvol in mGy) and dose length product $(\mathrm{DLP}$ in $\mathrm{mGy} \cdot \mathrm{cm})$ were documented for each patient.

\section{Quantitative Assessment}

Quantitative assessment of image quality was performed by quantifying the image noise (standard deviation [SD] of the CT number [Hounsfield units]) centrally at the vertebral bodies, disc spaces, and cerebrospinal fluid (CSF) using $20 \mathrm{~mm}^{2}$ regions of interest. These measurements were performed in the L2, L3 and L4 vertebral bodies; the $\mathrm{L} 2 / 3, \mathrm{~L} 3 / 4$ and $\mathrm{L} 4 / 5$ disc spaces, and the corresponding CSF spaces, unless visible pathology was identified at any of these levels. In those instances, measurements of regions of interest were taken at a lower level. Mean noise was calculated. These measurements were performed with both bone and soft tissue windows, yielding results for SIR and IMR for CTAP and SIR for L-spine CT. The contrast-to-noise ratio $(\mathrm{CNR})$ was measured using the formula:

$C N R=(C T$ number $[$ disc or bone $]-C T$ number $[C S F]) /$ Noise $(C S F)$

Although CNR measurements do not include the effect of image resolution and texture of noise, there was no other satisfactory quantitative measurement of image quality. ${ }^{19}$

\section{Qualitative Assessment}

Qualitative assessment was performed by two radiologists (a CT radiologist with 28 years of CT experience and a radiologist with 5 years of CT experience). The more experienced radiologist has an in-depth knowledge of IR techniques and has been working with these techniques for over 3 years. The other radiologist recruited in the study has lesser knowledge of IR techniques. Both independently read all CT lumbar spine images in a random order. The readers were required to allocate a score between 1 and 5 to the bone/CSF, intervertebral disc/CSF, and intervertebral disc/bone interfaces of the lumbar spine CT images, and from the different IR methods of CTAP. A score of 5 represented excellent demarcation of the interface and a score of 1 represented poor delineation of the interface. Median scores were obtained. Reading was performed on both bone and soft tissue windows. The readers were blinded to the patient details, clinical history, type of study, and original report.

\section{Inter-reader Variability}

Inter-reader variability was calculated using Cohen's kappa statistics: a $x$ value of $\leq 0.20$ indicated poor agreement and a $x$ value of $>0.80$ indicated good agreement.

\section{Data Analysis}

Data analysis was performed using Microsoft Excel ${ }^{\circledR}$ (Microsoft Corporation, Redmond, Washington [WA], 
US) and STATA 14 (StataCorp, College Station [TX], US). The IMR lumbar spine CT images were compared with SIR images of lumbar spine from CTAP and L-spine CT. Both quantitative and qualitative assessment results of the different lumbar spine reconstructions from CTAP and the L-spine CT were compared using the Mann-Whitney $U$ test. The results from the quantitative and qualitative assessment between the different IR techniques for CTAP were compared using paired $t$ tests. A $p$ value of $<0.05$ was considered significant.

\section{RESULTS}

A total of 91 cases (46 women and 45 men; mean age $61 \pm 19$ years, range 19-95) were selected for the CTAP group. Two cases with significant beam hardening artefact from spinal fusion screws were excluded from the L-spine CT group. After these two cases had been excluded, a total of 100 cases (53 women and 47 men; mean age $63 \pm 15$ years, range $31-83$ ) were included in the L-spine CT group.

Inter-reader agreements were high, $x=0.93$ and 0.85 , respectively for soft tissue and bony windows $(x$ value of $\leq 0.20$ indicated poor agreement and a $x$ value of $>0.80$ indicated good agreement).

The $\mathrm{kVp}$ for dedicated $\mathrm{L}$-spine $\mathrm{CT}$ was generally higher than that for CTAP. All L-spine CTs were routinely performed at $120 \mathrm{kVp}$ in our institution. In total, 77 CTAPs were performed at $100 \mathrm{kVp}$ whereas 14 CTAPs were performed at $120 \mathrm{kVp}$ based on patients' body weights $(100 \mathrm{kVp}$ for those weighing $<80 \mathrm{~kg}$ and $120 \mathrm{kVp}$ for those weighing $\geq 80 \mathrm{~kg}$ as our departmental CTAP scanning protocols). Mean mAs was significantly higher for L-spine CT $(386 \pm 180 \mathrm{mAs})$ than for CTAP
$(178 \pm 71 \mathrm{mAs})[\mathrm{p}<0.001]$. The scan range of L-spine CT was routinely from T12 to S2, which was shorter compared with CTAP, which was typically from the diaphragm (around T9 to T10 level) to the inguinal region (below the coccyx). Even with a shorter scanning range, DLP and CTDIvol were significantly higher for L-spine CT compared with CTAP: The mean DLP was significantly higher for L-spine CT $(774.1 \pm 363 \mathrm{mGy} \cdot \mathrm{cm})$ than for CTAP $(473.3 \pm 308 \mathrm{mGy} \cdot \mathrm{cm})[\mathrm{p}<0.001]$, and mean CTDIvol was significantly higher for L-spine CT (26.1 $\pm 12 \mathrm{mGy})$ than for CTAP $(8.6 \pm 5 \mathrm{mGy})[\mathrm{p}<0.001]$.

The time for image reconstruction using IMR was 3 to 5 minutes and about 2 minutes for SIR.

As demonstrated in Table 1, the SDs representing image noise varied widely between SIR and IMR. There were significant reductions in image noise in the lumbar spine vertebral bodies, intervertebral discs, and CSF on IMR compared with SIR lumbar spine images from the same CTAP (all $\mathrm{p}<0.001$ ). IMR lumbar spine images from CTAP also produced significantly less image noise than those of dedicated L-spine CT $(p<0.001)$. Further comparison made between the IMR lumbar spine images from the 14 CTAPs performed at $120 \mathrm{kVp}$ with L-spine CT (also performed at $120 \mathrm{kVp}$ ) again revealed statistically significant less noise on IMR spine images for this subgroup of CTAP at $120 \mathrm{kVp}(\mathrm{p}<0.001)$.

Qualitatively, IMR spine images from CTAP demonstrated significantly better interfaces between bone/CSF ( $p<0.001)$, disc/CSF $(p<0.001)$ and disc/ bone $(\mathrm{p}<0.001)$ than SIR images (Table 2). Compared with L-spine CT, SIR images were significantly inferior for all interfaces whereas IMR images were superior

Table 1. Image noise and contrast-to-noise ratio for computed tomography of the abdomen and pelvis (CTAP) and computed tomography of the lumbar spine (L-spine CT).

\begin{tabular}{|c|c|c|c|c|c|c|c|c|}
\hline & \multicolumn{6}{|c|}{ Image noise* (Hounsfield units) } & \multicolumn{2}{|c|}{ Contrast-to-noise ratio } \\
\hline & \multicolumn{3}{|c|}{ Bone window } & \multicolumn{3}{|c|}{ Soft tissue window } & \multirow[b]{2}{*}{$\begin{array}{l}\text { Disc/CSF } \\
\text { interface }\end{array}$} & \multirow[b]{2}{*}{$\begin{array}{c}\text { Bone/CSF } \\
\text { interface }\end{array}$} \\
\hline & Bone & Disc & CSF & Bone & Disc & CSF & & \\
\hline L-spine CT SIR & $76.3 \pm 51$ & $65.3 \pm 53$ & $63.0 \pm 60$ & $27.3 \pm 35$ & $17.2 \pm 8$ & $14.0 \pm 5$ & 4.9 & 2.1 \\
\hline CTAP SIR (100 kVp) & $184.5 \pm 38$ & $185 \pm 38$ & $173.7 \pm 38$ & $35.9 \pm 12$ & $32.5 \pm 14$ & $30.3 \pm 7$ & 2.2 & 1.1 \\
\hline CTAP SIR (120 kVp) & $149.0 \pm 37$ & $130.6 \pm 45$ & $122.2 \pm 41$ & $41.8 \pm 13$ & $32.6 \pm 16$ & $26.2 \pm 5$ & 2.4 & 1.0 \\
\hline CTAP IMR (100 kVp) & $17.9 \pm 9$ & $13.4 \pm 5$ & $12.4 \pm 4$ & $19.0 \pm 9$ & $14.0 \pm 4$ & $12.4 \pm 5$ & 4.7 & 12.8 \\
\hline CTAP IMR (120 kVp) & $23.3 \pm 10$ & $11.9 \pm 5$ & $13.4 \pm 5$ & $22.3 \pm 8$ & $11.3 \pm 4$ & $13.4 \pm 4$ & 4.8 & 11.3 \\
\hline
\end{tabular}

Abbreviations: CSF = cerebrospinal fluid; IMR = iterative model reconstruction; SIR = statistical iterative reconstruction.

* Data are presented as the mean \pm standard deviation of the region of interest. $p<0.001$ for all the values specified above. 
Table 2. Comparison of the quality of interfaces between computed tomography of the abdomen and pelvis (CTAP) and computed tomography of the lumbar spine (L-spine CT).*

\begin{tabular}{lccc}
\hline Interface & CTAP SIR & CTAP IMR & $\begin{array}{c}\text { L-spine CT } \\
\text { SIR }\end{array}$ \\
\hline Bone/cerebrospinal fluid & $3(2-4)$ & $5(3-5)$ & $4(2-5)$ \\
Disc/cerebrospinal fluid & $1(1-3)$ & $4(2-5)$ & $3(1-4)$ \\
Disc/bone & $3(2-4)$ & $5(3-5)$ & $5(3-5)$ \\
\hline
\end{tabular}

Abbreviations: IMR = iterative model reconstruction; $\mathrm{SIR}=$ statistical iterative reconstruction.

* Data are presented as median (range). $p<0.001$ for all the values specified above.

to L-spine CT for bone/CSF $(\mathrm{p}<0.001)$ and disc/CSF $(\mathrm{p}<0.001)$ interfaces and of equal quality in delineating disc/bone interface (Figures 1-4).

\section{DISCUSSION}

There is an increasing tendency of generating multiplanar reconstructed spinal images from CTAP examinations, particularly in the trauma setting. ${ }^{4}$ This is primarily because they are more sensitive in detecting fractures compared with plain radiographs and avoid subjecting patients to a second CT spine study. ${ }^{4}$ Similarly, in a nontrauma setting, especially oncology patients and patients with pyrexia of unknown origin, spinal reconstructions are routinely performed to assist in the detection of bone, paravertebral, and disc pathologies. ${ }^{20}$

Image noise levels were significantly higher for reconstructed lumbar spine images from CTAP compared with L-spine CT, when both were reconstructed using SIR. As a result, the interfaces between bone, disc, CSF, and soft tissues were less well defined in the reconstructed spinal images from CTAP (Figure 2) due to increased image noise, making detection of subtle fractures and disc pathology more difficult. However, the reduction in image noise on L-spine CT was at the expense of patients' radiation exposure.

Image noise in reconstructed lumbar spine images from CTAP can be improved by applying IMR. Our study confirms that IMR is superior to SIR for both bone and soft tissue assessment in lumbar spine reconstructions from CTAP and those from routine L-spine CT due to reduction of image noise. This was agreed upon by both our radiologists of different years of experience as evident from our high inter-reader agreement. Apart from image noise and radiation dose reduction, IMR is also capable of improving low-contrast target visibility, which is important when detecting disc pathology and small fractures in osteoporotic bone. Therefore, using IMR for lumbar spine reconstruction from CTAP may negate the need for performing a separate L-spine CT in trauma patients who have already undergone a CTAP. As a consequence of image noise reduction and improved low-contrast target visibility, traumatic (fractures) and non-traumatic (disc bulges, metastases) pathologies may become more conspicuous and better appreciated on IMR images. This would be cost-effective in providing a timely diagnosis without need for a second scan.

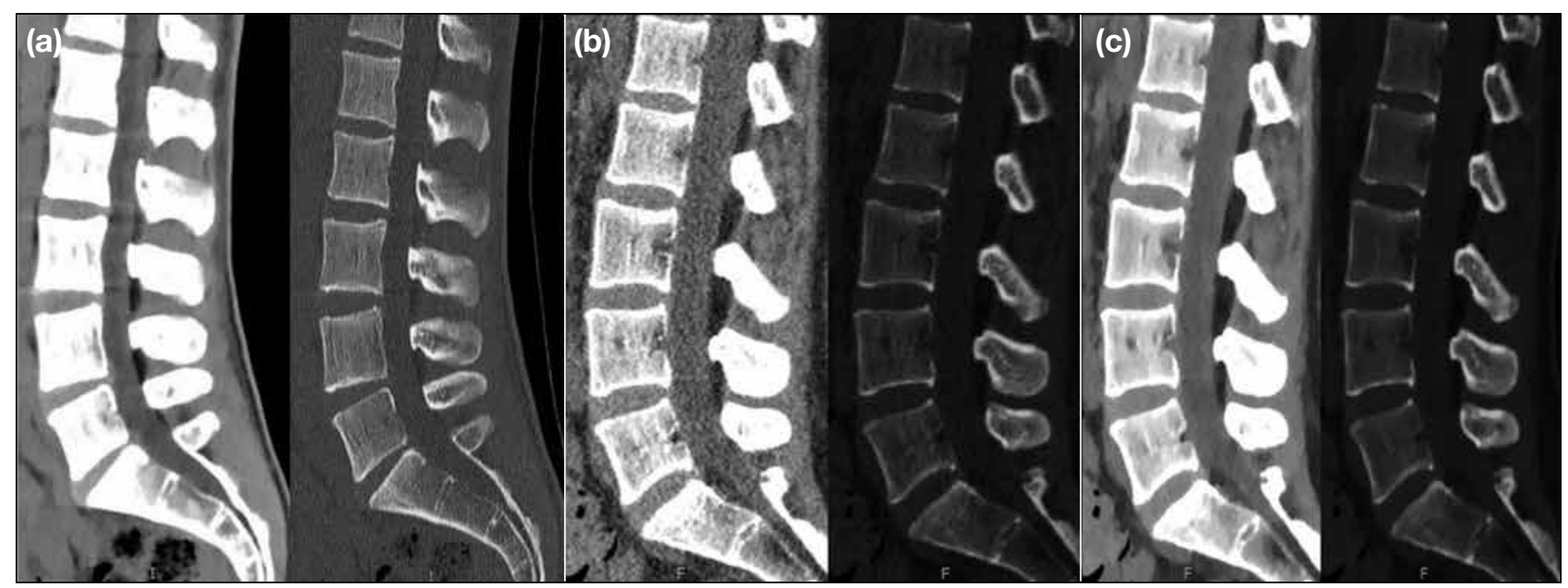

Figure 1. (a) Computed tomography image of the lumbar spine (L-spine CT). (b) Statistical iterative reconstruction (SIR) of computed tomography of the abdomen and pelvis (CTAP) images and (c) iterative model reconstruction (IMR) of CTAP images in soft tissue and bone windows. The IMR image in (c) rendered less image noise and better low-contrast target visibility such that the interfaces between bone, disc, and cerebrospinal fluid (CSF) were better delineated compared with those in CTAP in (b) and were also better in disc/CSF and bone/ CSF interfaces compared with those of SIR images of dedicated L-spine CT in (a). 

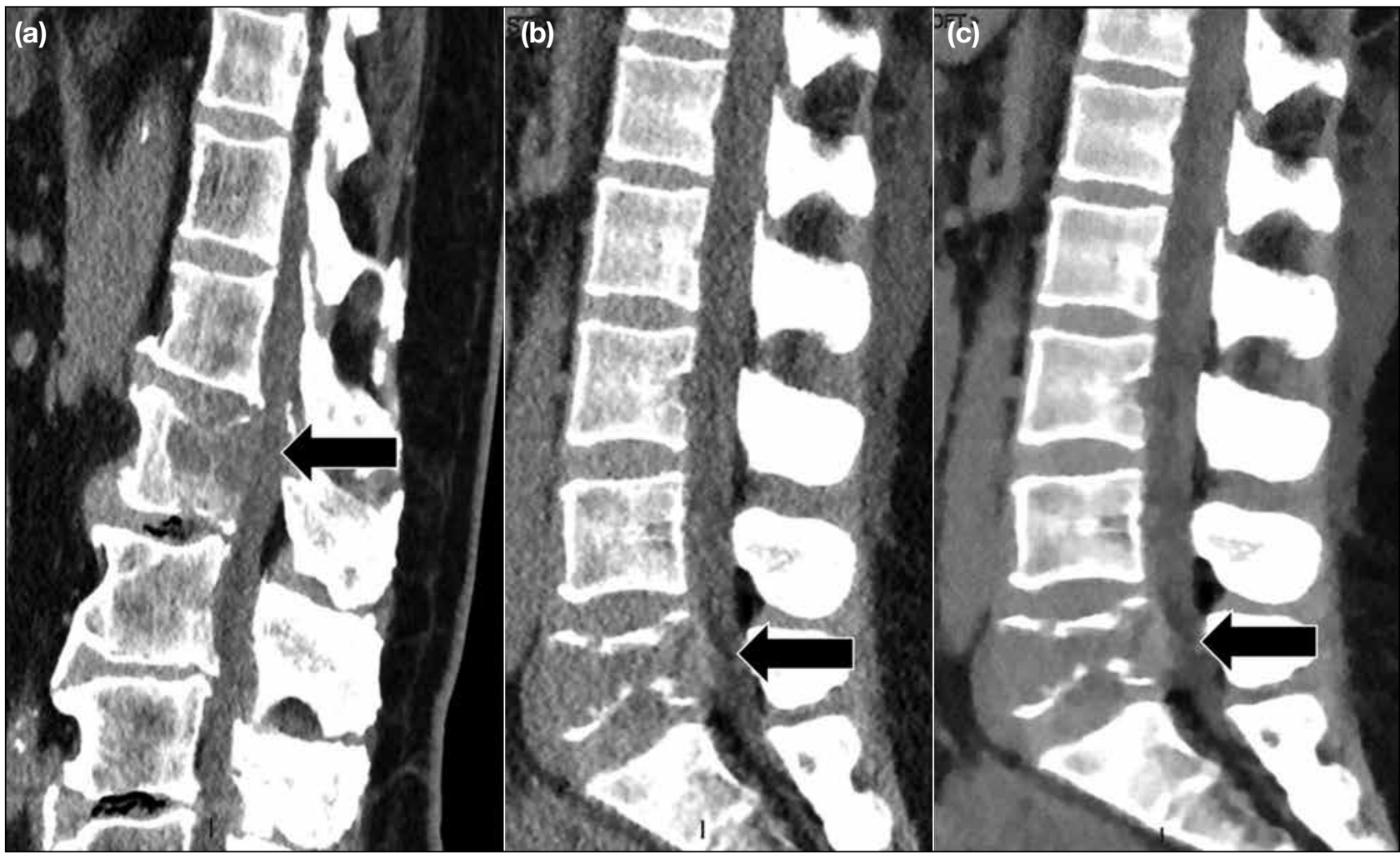

Figure 2. (a) Bone metastases (indicated by black arrows) on computed tomography image of the lumbar spine (L-spine CT) using statistical iterative reconstruction (SIR) of an adult patient. Computed tomography images of the abdomen and pelvis (CTAP) of a different patient using (b) SIR and (c) iterative model reconstruction (IMR). The IMR image in (c) shows better interfaces between metastasis, bone, and cerebrospinal fluid compared with those of SIR images from (b) CTAP and dedicated (a) L-spine CT.

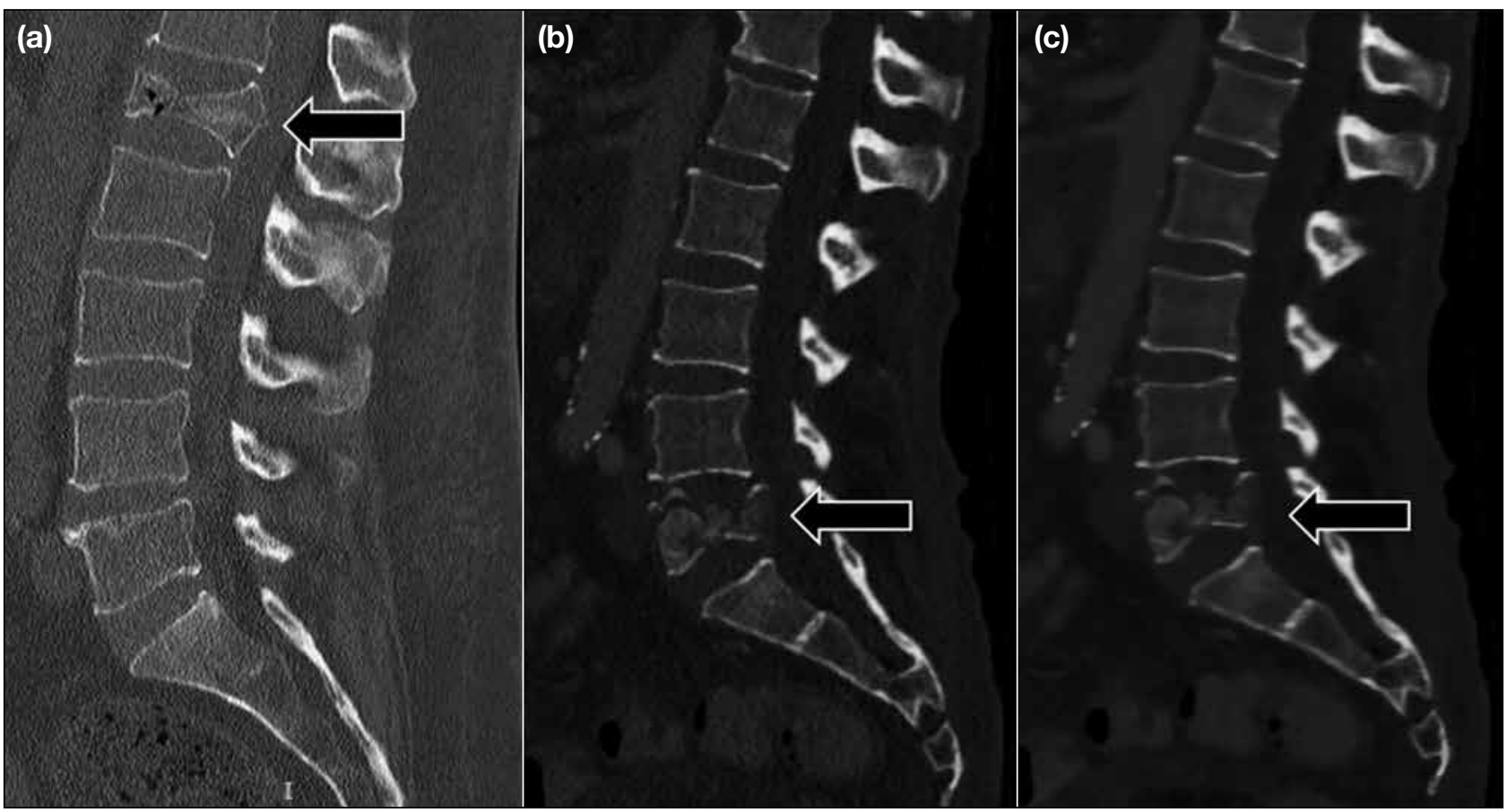

Figure 3. (a) Fractures (indicated by black arrows) on computed tomography image of the lumbar spine (L-spine CT) using statistical iterative reconstruction (SIR) of an adult patient. Computed tomography images of the abdomen and pelvis (CTAP) of another adult patient using (b) SIR and (c) iterative model reconstruction (IMR). The fracture lines and fragments are better defined on the IMR image in (c). 


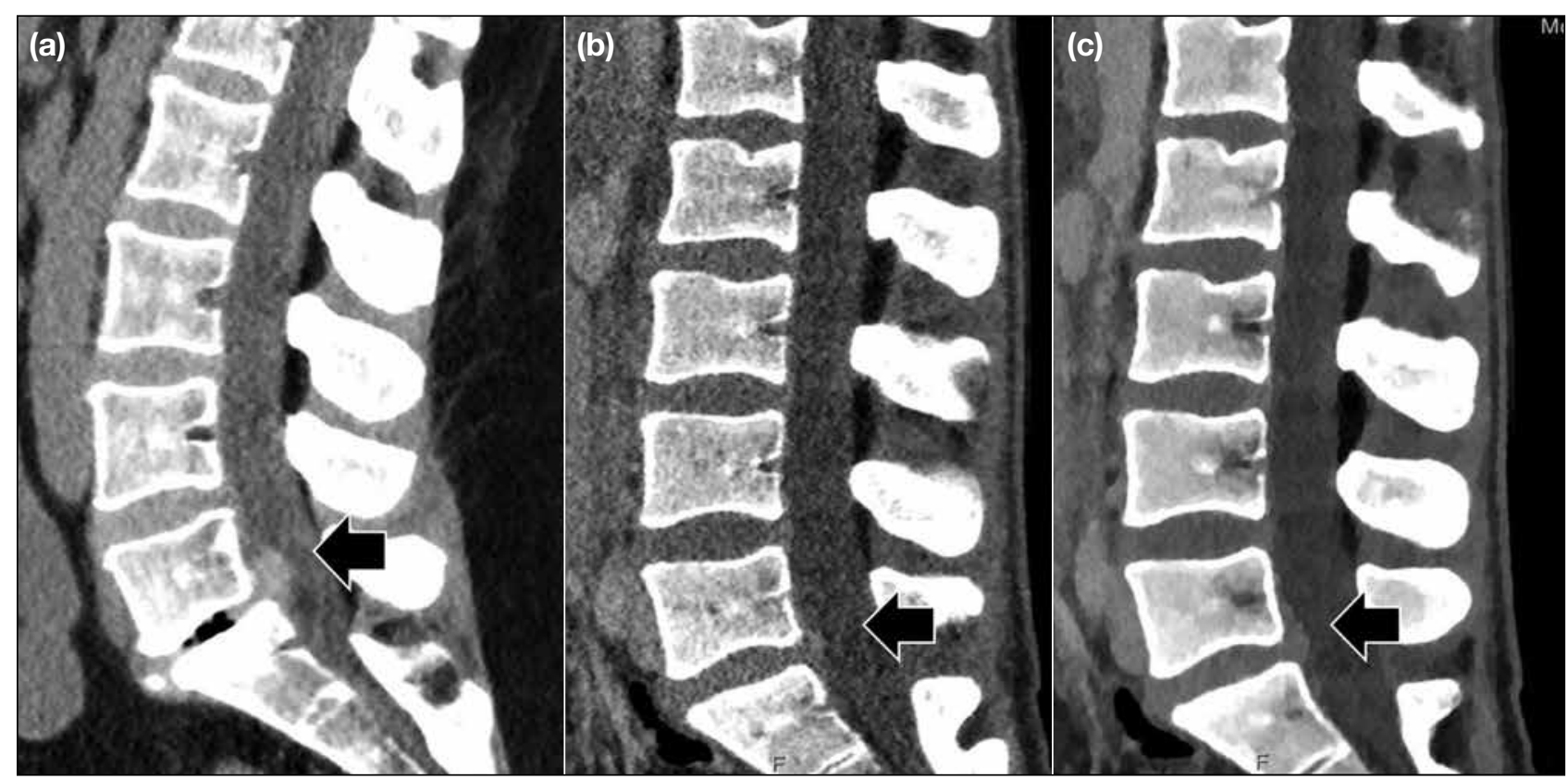

Figure 4. (a) Spinal canal stenosis (indicated by black arrows) due to disc protrusion on computed tomography of the lumbar spine using statistical iterative reconstruction of an adult patient. Computed tomography images of the abdomen and pelvis of another adult patient using (b) statistical iterative reconstruction and (c) iterative model reconstruction (IMR). The interface between disc and cerebrospinal fluid was far better visualised on IMR image (c) due to the reduction of noise and improved low subject contrast visibility.

However, IMR CTAP images can produce a 'plastic' appearance or 'blotchy' texture as described by our readers as well as radiologists in other studies. ${ }^{18}$ The learning curve for interpreting these images will vary for different individuals and the degree of experience and confidence plays an important role. The benefits are more pronounced when radiologists are more familiar with the IMR CT image appearance. Our radiologists, with different lengths of experience, both adapted quite quickly to the change in CT appearance. Other radiologists from different settings and regions may have different opinions and experiences on the reconstructed images.

Radiation dose is generally higher in L-spine CT compared with routine CTAP despite the shorter scanning range due to two main reasons. First, a higher $\mathrm{mAs}$ is required to generate better image quality for the bone and soft tissue details, resulting in a linear increase in radiation dose. ${ }^{21}$ Second, utilisation of higher $\mathrm{kVp}$ in order to improve image quality of the bones causes a dose increase approximately proportional to the square of the change in tube voltage. ${ }^{21}$ Multiple other factors, such as length of time of acquisition, may affect the image quality, which could not be standardised in this study due to its retrospective nature.
Limitations of this study included small number of cases. Because this was a retrospective study, patients did not undergo both CTAP and L-spine CT, making direct comparisons in the same patient impossible. Furthermore, the patients were scanned at different scanning parameters such as $\mathrm{kVp}$ which might affect the CNR. Despite this, the small subgroup CTAPs acquired at $120 \mathrm{kVp}$ confirmed improved image quality of the lumbar spine with IMR. Future prospective studies with a larger patient cohort, a larger group of radiologists, as well as dedicated qualitative assessment of various lumbar spine pathologies using different IR techniques, would be helpful to confirm the benefits of IMR in the reconstructed lumbar spine images from CTAP.

\section{CONCLUSION}

IMR lumbar spine images from CTAP were shown to produce less image noise and better low-contrast target detectability, and therefore, resulted in better image quality even when compared with dedicated CT lumbar spine images. These IMR lumbar spine images provided better interface details compared with that of dedicated SIR L-spine CT examinations. This may negate the need for performing dedicated lumbar spine $\mathrm{CT}$ in some patients and therefore, aids in the timely diagnosis, which is important in trauma and oncology settings. It 
may also help streamline the workflow in a busy tertiary imaging centre.

\section{REFERENCES}

1. The 2007 recommendations of the International Commission on Radiological Protection. ICRP publication 103. Ann ICRP. 2007;37:1-332.

2. Tamm EP, Rong XJ, Cody DD, Ernst RD, Fitzgerald NE, Kundra V. Quality initiatives: CT radiation dose reduction: how to implement change without sacrificing diagnostic quality. Radiographics. 2011;31:1823-32.

3. Soto JA, Anderson SW. Multidetector CT of blunt abdominal trauma. Radiology. 2012;265:678-93.

4. Carter B, Griffith B, Mossa-Basha F, Zintsmaster SA, Patel S, Williams TR, et al. Reformatted images of the thoracic and lumbar spine following CT of chest, abdomen, and pelvis in the setting of blunt trauma: are they necessary? Emerg Radiol. 2015;22:373-8.

5. Gross EA. Computed tomographic screening for thoracic and lumbar fractures: is spine reformatting necessary? Am J Emerg Med. 2010;28:73-5.

6. Lee SY, Landis MS, Ross IG, Goela A, Leung AE. Extraspinal findings at lumbar spine CT examinations: prevalence and clinical importance. Radiology. 2012;263:502-9.

7. Lee K, Beveridge T, Sanagou M, Thomas P. Updated Australian diagnostic reference levels for adult CT. J Med Radiat Sci. 2020;67:5-15.

8. Deák Z, Grimm JM, Treitl M, Geyer LL, Linsenmaier U, Körner M, et al. Filtered back projection, adaptive statistical iterative reconstruction, and a model-based iterative reconstruction in abdominal CT: an experimental clinical study. Radiology. 2013;266:197-206.

9. Löve A, Siemund R, Höglund P, Van Westen D, Stenberg L, Petersen $\mathrm{C}$, et al. Hybrid iterative reconstruction algorithm in brain CT: a radiation dose reduction and image quality assessment study. Acta Radiol. 2014;55:208-17.

10. Willemink MJ, de Jong PA, Leiner T, de Heer LM, Nievelstein RA, Budde RP, et al. Iterative reconstruction techniques for computed tomography Part 1: technical principles. Eur Radiol. 2013;23:162331.

11. Hara AK, Paden RG, Silva AC, Kujak JL, Lawder HJ, Pavlicek W.
Iterative reconstruction technique for reducing body radiation dose at CT: feasibility study. AJR Am J Roentgenol. 2009;193:764-71.

12. Gatewood MO, Grubish L, Busey JM, Shuman WP, Strote J. The use of model-based iterative reconstruction to decrease ED radiation exposure. Am J Emerg Med. 2015;33:559-62.

13. Klink T, Obmann V, Heverhagen J, Stork A, Adam G, Begemann P. Reducing CT radiation dose with iterative reconstruction algorithms: the influence of scan and reconstruction parameters on image quality and CTDIvol. Eur J Radiol. 2014;83:1645-54.

14. Kordolaimi SD, Saradeas I, Ploussi A, Pantos I, Argentos S, Efstathopoulos EP. Introduction of an effective method for the optimization of CT protocols using iterative reconstruction algorithms: comparison with patient data. Am J Roentgenol. 2014;203:W434-9.

15. Willemink MJ, Leiner T, de Jong PA, de Heer LM, Nievelstein RA, Schilham AM, et al. Iterative reconstruction techniques for computed tomography part 2: initial results in dose reduction and image quality. Eur Radiol. 2013;23:1632-42.

16. Park SB, Kim YS, Lee JB, Park HJ. Knowledge-based iterative model reconstruction (IMR) algorithm in ultralow-dose CT for evaluation of urolithiasis: evaluation of radiation dose reduction, image quality, and diagnostic performance. Abdom Imaging. 2015;40:3137-46.

17. Löve A, Olsson ML, Siemund R, Stålhammar F, BjörkmanBurtscher IM, Söderberg M. Six iterative reconstruction algorithms in brain CT: a phantom study on image quality at different radiation dose levels. Br J Radiol. 2013;86:20130388.

18. Zhang M, Qi W, Sun Y, Jian Y, Liu X, Hong N. Screening for lung cancer using sub-millisievert chest $\mathrm{CT}$ with iterative reconstruction algorithm: image quality and nodule detectability. $\mathrm{Br} \mathrm{J}$ Radiol. 2018;91:20170658.

19. Christianson O, Chen JJ, Yang Z, Saiprasad G, Dima A, Filliben JJ, et al. An improved index of image quality for task-based performance of CT iterative reconstruction across three commercial implementations. Radiology. 2015;275:725-34.

20. Meyer CA, Vagal AS, Seaman D. Put your back into it: pathologic conditions of the spine at chest CT. Radiographics. 2011;31:142541.

21. Kalra MK, Maher MM, Toth TL, Hamberg LM, Blake MA, Shepard JA, et al. Strategies for CT radiation dose optimization. Radiology. 2004;230:619-28. 